\title{
Intelligent GPS receiver for robust carrier phase tracking in kinematic environments
}

\author{
W.-L. Mao, H.-W. Tsao and F.-R. Chang
}

\begin{abstract}
Carrier phase measurement is essential for high accuracy positioning in mobile Global Positioning System (GPS) applications. For GPS receiver design, a narrow loop noise bandwidth is desirable to reduce the phase jitter due to thermal noise. However, it deteriorates the capability of tracking loops and may result in cycle slips. Based on an adaptive bandwidth criterion, a new design procedure for intelligent GPS receivers is presented to improve carrier phase tracking in the presence of highly kinematic environments. A fuzzy logic controller (FLC), which uses the carrier phase and frequency errors as input data, is first employed to provide rapid and accurate control of digital phase-locked loops (DPLL) in the transient and steady states. When the phase error or frequency error is large, the intelligent carrier loop increases the loop bandwidth adaptively and performs fast locking. Once the tracking errors are reduced, this tracking loop decreases the loop bandwidth and improves ranging accuracy. By utilising the highest dynamic stress information, the FLC loop is developed to offer several advantages over traditional methods in acquisition limitations, these being: wider lock range $(150 \mathrm{~Hz})$ and pull-in range $(400 \mathrm{~Hz})$; faster pull-in speed; and larger frequency ramp range $(412 \mathrm{~Hz} / \mathrm{s})$. Simulation results demonstrate that the proposed fuzzy-based receiver does achieve a shorter settling time and broader acquisition range than conventional tracking loops while preventing the occurrence of cycle skipping.
\end{abstract}

\section{Introduction}

The Global Positioning System (GPS) receiver has become a commonly used navigation instrument for aircraft precision approaches, missile systems, automated vehicle guidance, and other applications. Making use of carrier phase information can provide the highest surveying measurements within a millimetre level of accuracy. However, robust carrier phase tracking is still a challenging task for GPS when the receivers undergo significant dynamics [1]. To obtain an accurate estimate of phase information, narrow loop noise bandwidth is often utilised in carrier loops to decrease the thermal noise and extract the precise phase of the carrier signal. There is a price to pay, however, and that price is dynamic performance. This can lead to a serious degradation in PLL acquisition characteristics and result in a poor pull-in (or capture) capability. Under highly dynamic environments, the carrier phases cannot be tracked promptly by this conventional digital phase-locked loop (DPLL), and this failing will increase the probability of cycle skipping. Once cycle slips occur, several measurement epochs will be missed, and a timeconsuming ambiguity search procedure needs to be

\section{(C) IEE, 2004}

IEE Proceedings online no. 20040188

doi: 10.1049/ip-rsn:20040188

Paper first received 12th November 2002 and in revised form 30th October 2003

W.-L. Mao is with the Department of Electrical Engineering, Mingchi Institute of Technology, 84 Gungjuan Rd. Taishan, Taipei Hsien, Taiwan 243, Republic of China

H.-W. Tsao and F.-R. Chang are with the Graduate Institute of Electronics Engineering and Department of Electrical Engineering, National Taiwan University, No. 1, Sec. 4, Roosevelt Rd., Taipei 106, Taiwan, Republic of China restarted. To overcome this notable contradiction, some new adaptive-bandwidth DPLLs are necessary to improve the phase tracking capacity and positioning accuracy simultaneously.

Few researchers have investigated the GPS receiver design in a variety of mobile environments. Simon and El-Sherief [1] proposed a fuzzy PLL estimation filter for missile navigation. Gradient-descent and genetic algorithms were used to optimise the fuzzy estimators. However, their tracking model needed a priori known specific trajectory (including nominal satellite-to-missile range, velocity and acceleration) to train the fuzzy PLL architecture, and thus it was not a suitable method for practical receiver design. Jwo [2] presented an optimal estimator for bandwidth determination in dynamic environments based on a linear Kalman filter. The optimal noise bandwidths were obtained for a linear PLL model in the steady state. However, if the receivers operate in kinematic circumstances, the linear model assumption no longer holds. Furthermore, the PLL may lose tracking in the transient process and never return to the synchronisation operation [3]. To date, no studies have attempted to design an intelligent receiver in which the motion direction is subject to very large dynamics. It is an important problem, as GPS receivers are utilised in various consumer and aerospace applications.

This paper presents a new GPS receiver architecture capable of varying the loop bandwidth in accordance with signal dynamics to provide robust carrier phase tracking. In general, the mobile carrier phase signal is comprised of five components, these being: (i) a phase offset; (ii) a sinusoidal phase jitter; (iii) a frequency offset; (iv) a frequency ramp offset; and (v) a jerk offset. On the basis of a vehicle's maximum dynamic stress, the loop order of the PLL and the corresponding acquisition limitations, including lock range, frequency ramp range, or jerk range, can be determined. The soft-computing fuzzy logic technique can co-operate with the digital loop filter to accomplish intelligent control of 
noise bandwidth in an on-line fashion. The carrier phase and frequency errors periodically estimated by a phase discriminator (PD) and a frequency discriminator (FD) are used as two inputs for the fuzzy logic controller (FLC). When the absolute value of the phase error or frequency error is increasing under conditions of high acceleration, the FLC produces an adaptive gain to the digital loop filter and varies the tracking loop bandwidth automatically. Once the tracking errors approach zero in the steady state, the loop bandwidth shrinks and returns to a narrowband DPLL. By tuning the control rules and membership functions iteratively, the intelligent FLC can regulate the DPLL swiftly and improve the acquisition capability. Based on this adaptive bandwidth-control algorithm, our FLC carrier loop is elaborated to provide: (i) a wider lock range and pull-in range; (ii) a faster pull-in process; and (iii) a larger frequency ramp range in a wide variety of kinematic environments.

\section{Kinematic system descriptions}

\subsection{Carrier phase model}

Figure 1 illustrates a simplified block diagram of the baseband signal processor of a GPS receiver channel. The received discrete time observation sample can be modelled as

$$
r\left(i T_{s}\right)=\sqrt{2 P} D\left[i T_{s}-\tau\right] C A\left[i T_{s}-\tau\right] \cos \left[\omega_{I F} i T_{s}+\theta\left(i T_{s}\right)\right]+n_{i}
$$

where $P$ is the average power of the received signal, $D[\cdot]$ is the binary data (50 bps) sequence, $C A[\cdot]$ is the Gold code C/A sequence, $T_{s}=1 / f_{s}$ is the sample period, $\tau$ is the code transmission time delay with respect to the GPS system time, $\omega_{I F}$ is the IF carrier radian frequency, $\theta\left(i T_{s}\right)$ is the unknown carrier phase to be estimated, and $n_{i}$ is the received noise modelled as additive white Gaussian noise (AWGN) with a two-sided power spectral density of $N_{0} / 2 \mathrm{~W} / \mathrm{Hz}$.

The relative motion of satellites and vehicles has significant effects on the phase and frequency tracking of GPS receivers. The kinematic carrier phase $\theta\left(i T_{s}\right)$ mainly includes five components: $(a)$ phase offset, $(b)$ sinusoidal phase jitter, $(c)$ frequency offset, $(d)$ frequency ramp offset and (e) jerk (frequency acceleration) offset. A mathematical model for the carrier phase at the sampling instants can be expressed as

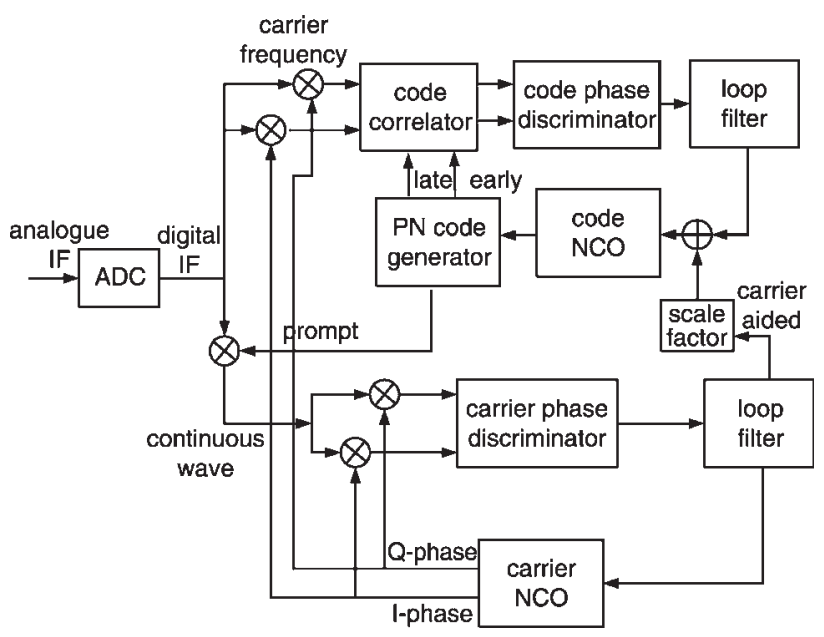

Fig. 1 Simplified GPS baseband block diagram

$$
\begin{aligned}
\theta\left(i T_{s}\right)= & \theta_{0}+\sum_{j=1}^{J} A_{j} \sin \omega_{j}\left(i T_{s}\right)+\omega_{0}\left(i T_{s}\right) \\
& +\frac{1}{2} \alpha_{0}\left(i T_{s}\right)^{2}+\frac{1}{6} J_{0}\left(i T_{s}\right)^{3}
\end{aligned}
$$

where $\theta_{0}(\mathrm{rad})$ is a constant phase difference between the satellite and receiver. The phase jitter arises as the response of vibration behaviour of the kinematic vehicles on the fly and is typically modelled as a sum of sinusoidal waveforms with frequencies $\omega_{j}$ and amplitudes $A_{j}$. $\omega_{0}(\mathrm{rad} / \mathrm{s})$ is the amount of Doppler frequency fluctuations. $\alpha_{0}\left(\mathrm{rad} / \mathrm{s}^{2}\right)$ and $J_{0}\left(\mathrm{rad} / \mathrm{s}^{3}\right)$ are the frequency $\mathrm{ramp}$ and frequency acceleration of the highly dynamic users. The frequency ramp caused by the satellite motion (as much as $1 \mathrm{~Hz} / \mathrm{s}$ ) is usually small in GPS, so we will consider only the motion of vehicles [4]. We assume that the user has an acceleration of $a_{r}\left(\mathrm{~m} / \mathrm{s}^{2}\right)$, and the equivalent Doppler frequency ramp is then

$$
\alpha_{0}=\left(\frac{\omega_{c}}{c}\right) a_{r}\left(\mathrm{rad} / \mathrm{s}^{2}\right)
$$

where $c$ is the speed of light and $\omega_{c}(\mathrm{rad} / \mathrm{s})$ is the GPS L1 carrier radian frequency $\left(=2 \pi \times 1575.42 \times 10^{6} \mathrm{rad} / \mathrm{s}\right)$. As an example, for a high-speed airplane with acceleration of $7 G(G=$ gravitational acceleration with a value of $\left.9.8 \mathrm{~m} / \mathrm{s}^{2}\right)$, the corresponding Doppler frequency ramp $\alpha_{D}$ is $2263.5 \mathrm{rad} / \mathrm{s}^{2}(=360 \mathrm{~Hz} / \mathrm{s})$. The $Z$-transform of the phase signal is given by

$$
\begin{aligned}
\theta(z)= & \frac{\theta_{0} z}{z-1}+\sum_{j=1}^{J} \frac{A_{j} \sin \omega_{j} T_{s} z}{z^{2}-2 \cos \omega_{j} T_{s} z+1}+\frac{\omega_{0} T_{s} z}{(z-1)^{2}} \\
& +\frac{\alpha_{0} T_{s}^{2} z(z+1)}{2(z-1)^{3}}+\frac{J_{0} T_{s}^{3} z\left(z^{2}+4 z+1\right)}{6(z-1)^{4}}
\end{aligned}
$$

For this great diversity of phase components, an intelligent receiver design is required to acquire the desired frequency and frequency ramp offset promptly and without losing lock.

\subsection{Cycle slips}

Because of the large dynamics and phase noise in satellite communication channels, the carrier tracking errors will change rapidly and result in cycle skipping. Since a phase discriminator cannot recognise the difference between $\theta_{e}$ and $\theta_{e}+2 n \pi$ ( $n$ is an integer), synchronisation failure may occur. This phenomenon is called cycle slipping, i.e. jumps of the carrier phase by an integer multiple of periods. Once cycle slips happen, they degrade the accuracy of the carrier phase range and the position message obtained from such carrier phase observables. Restarting the carrier phase ambiguity process is a time and computation intensive task and should be avoided for as long as possible.

In the carrier loop design, a tradeoff between two contradicting demands is always involved. The thermal noise jitter is sensitive to the loop noise bandwidth. Decreasing loop noise bandwidth can prevent loss of lock due to thermal noise. However, it will also reduce the ability of the loop to track the Doppler-shifted carrier phase signal induced by user dynamics. In typical GPS applications, the tracking loop bandwidths $B_{n}$ are usually designed to be in the range $5-15 \mathrm{~Hz}$ [5]. The acquisition parameters of such a second-order PLL [3, 6] can be obtained approximately as: 


$$
\begin{aligned}
\omega_{n} & =2 B_{n}\left(\zeta+0.25 \zeta^{-1}\right)^{-1} \\
\omega_{L} & \approx 2 \xi \omega_{n} \\
\omega_{P O} & \approx 1.8 \omega_{n}(\xi+1) \\
\alpha_{P O} & \approx \omega_{n}^{2}
\end{aligned}
$$

where $\omega_{n}(\mathrm{rad} / \mathrm{s})$ is the natural frequency and $\xi$ is the damping ratio. $\omega_{L}$ is the lock range in which the PLL can lock within a single cycle, $\omega_{P O}$ is the pull-out range and $\alpha_{P O}$ is the frequency ramp range. The loop can regain lock for frequency $|\omega|<\omega_{P O}$ and frequency ramp $|\alpha|<\alpha_{P O}$. Taking $\xi=0.707$, the pull-out range is $\omega_{P O} \approx 87 \mathrm{rad} / \mathrm{s}$ $(=13.8 \mathrm{~Hz})$ and the frequency ramp range is $\alpha_{P O} \approx$ $800 \mathrm{rad} / \mathrm{s}^{2}(=127.3 \mathrm{~Hz} / \mathrm{s})$. It is difficult to maintain lock for such a narrowband PLL in the presence of the severe Doppler effects and acceleration dynamics.

The thermal noise is another possible cause of cycle slipping in PLL. The one-sigma thermal noise jitter [7] has been derived:

$$
\sigma_{R}^{2}=\frac{B_{n}}{C N R}\left[1+\frac{1}{2 T \times C N R}\right]
$$

where $\sigma_{R}(\mathrm{rad})$ is the tracking error standard deviation, $\mathrm{T}(\mathrm{s})$ is the predetection integration time (PIT), and CNR is the carrier-to-noise ratio, which typically ranges from 35 to $55 \mathrm{dBHz}$ [5] in GPS. In nominal ranges of CNR, the thermal noise can always be considered insignificant compared to the phase error described above. If we assume $B_{n}=10 \mathrm{~Hz}$ and $T=1 \mathrm{~ms}$, the three-sigma value $\left.\left(3 \sigma_{R}\right)\right)$ is $0.017-0.182 \mathrm{rad}$. It is obvious that cycle slipping due to high dynamic trajectory becomes the dominant factor during the transient periods.

\section{Proposed intelligent receiver}

When the SV code and frequency have been successfully replicated during the search process, the carrier loop is activated to synchronise the incoming phase signal. Figure 2 demonstrates the block diagram of our proposed GPS carrier-tracking loop. This system is composed of two baseband correlators, a phase discriminator (PD), a frequency discriminator (FD), a fuzzy logic controller (FLC), a loop filter, and a numerical controlled oscillator (NCO). The PD and FD use both in-phase (I) and quadrature-phase $(\mathrm{Q})$ samples to produce the carrier phase and frequency errors. The intelligent loop filter, consisting of the FLC and digital filter, achieves adaptive bandwidth adjustment to improve the acquisition capacity and ranging performance.

\subsection{Phase/ frequency discriminator}

The received sequence $r\left(i T_{s}\right)$ is mixed with the local prompt code and $I / Q$ replica carrier signals for carrier synchronisation. After the integration and dump processes, the outputs of I- and Q-phase accumulators become:

$$
\begin{aligned}
& I(k T)=\frac{A}{\sqrt{2}} \cos [\theta(k T)-\hat{\theta}(k T)]+n_{I, k} \\
& Q(k T)=\frac{A}{\sqrt{2}} \sin [\theta(k T)-\hat{\theta}(k T)]+n_{Q, k}
\end{aligned}
$$

where $A=2 \sqrt{P} M D(k T) \sin c(\Delta \omega T / 2) R(\tau-\hat{\tau}) \quad$ is the integrated amplitude of $I / Q$ phase components, $\sin c(x)=\sin x / x, R(\cdot)$ is the autocorrelation function of C/A codes, $\Delta \omega=\left(\theta_{k}-\theta_{k-1}\right) / T$, and $M=T / T_{s}$ is the number of samples summed together to update the loop. $\theta(k T)$ and $\hat{\theta}(k T)$ are the carrier phases of the received signal and local carrier signal. $n_{I, k}$ and $n_{Q, k}$ are I- and Q-phase baseband noise components due to input noise $n_{i}$.

The PD and FD measure the phase and frequency differences between the input signal and the NCO output signal. The phase and frequency errors can be computed using arc-tangent functions as given by:

$$
\begin{gathered}
\theta_{e}(k T)=\tan ^{-1}\left[\frac{Q_{k}}{I_{k}}\right]=\phi(k T)+n_{\theta, k} \\
\omega_{e}(k T)=\tan ^{-1}\left[\frac{C P_{k}}{D P_{k}}\right]=[\phi(k T)-\phi(k T-T)]+n_{\omega, k}
\end{gathered}
$$

with:

$$
\begin{gathered}
\phi_{k}=\theta_{k}-\hat{\theta}_{k} \\
D P_{k}=I_{k} \times I_{k-1}-Q_{k} \times Q_{k-1} \\
C P_{k}=I_{k-1} \times Q_{k}-I_{k} \times Q_{k-1}
\end{gathered}
$$

where $\theta_{e}(k T) \in[-\pi, \pi]$ is the carrier phase error with input noise disturbance $n_{\theta, k}$, and $\omega_{e}(k T) \in[-\pi, \pi]$ is the phase difference offset proportional to the rotation rate of a signal phase with noise disturbance $n_{\omega, k} . D P_{k}$ is the dot-product vector and $C P_{k}$ is the cross-product vector derived form the successive baseband signal vectors $\left[I_{k}, Q_{k}\right]$ and $\left[I_{k-1}, Q_{k-1}\right]$.

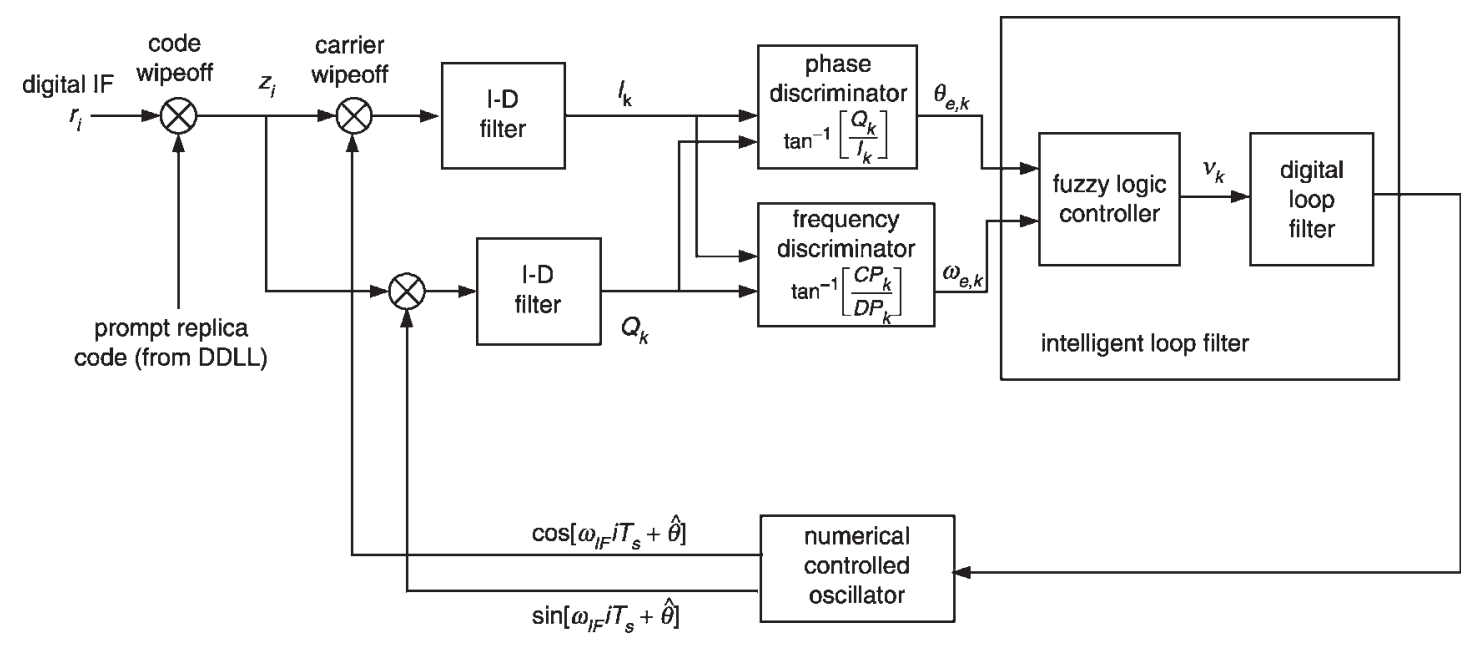

Fig. 2 Proposed intelligent GPS carrier tracking loop 


\subsection{Fuzzy logic controller}

We here develop a new carrier-tracking loop based on the digital PLL architecture, in which a fuzzy logic controller is designed according to the vehicle's dynamics. The equivalent baseband model of an FLC third-order loop is shown in Fig. $3 a$. Fuzzy control provides a means of converting a control strategy composed of a set of linguistic rules into an automatic control strategy. This technique requires only moderate computational complexity compared to conventional schemes, while tracking the phase and frequency fluctuations effectively and rapidly. We employ two input variables and one output variable in the FLC design. The fuzzy inputs are:

$$
\begin{aligned}
& x_{1, k}=\theta_{e, k} \\
& x_{2, k}=\omega_{e, k}
\end{aligned}
$$

where $\theta_{e, k}$ and $\omega_{e, k}$ are the phase error and frequency error determined from (8), respectively. The fuzzy output variable $v_{k}$ gives the adaptive driving gain (error) to the digital filter in the loop. Both FLC and loop filter join together as an intelligent loop filter, which widens and
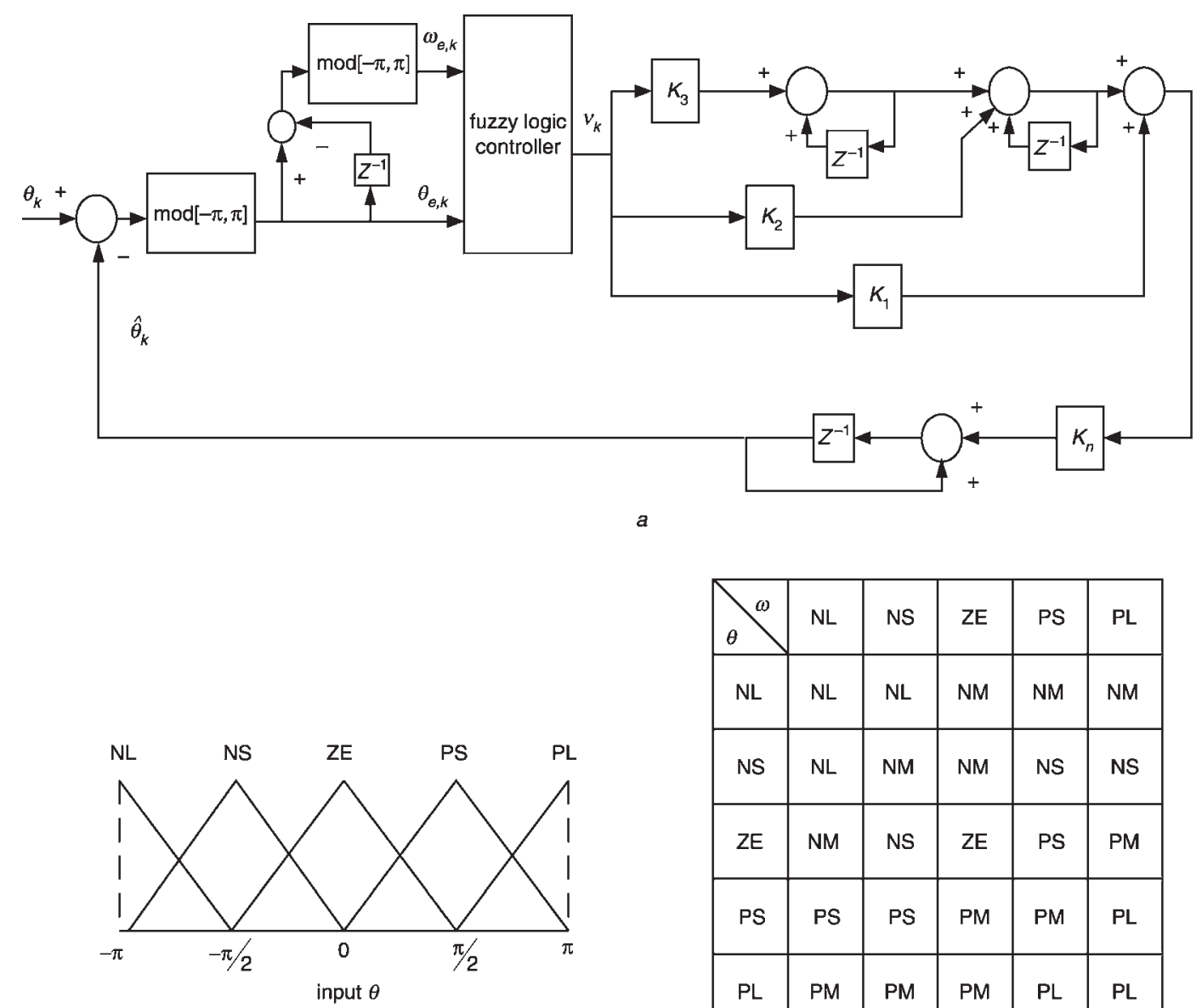

\begin{tabular}{|c|c|c|c|c|c|}
\hline$\theta$ & NL & NS & ZE & PS & PL \\
\hline NL & NL & NL & NM & NM & NM \\
\hline NS & NL & NM & NM & NS & NS \\
\hline ZE & NM & NS & ZE & PS & PM \\
\hline PS & PS & PS & PM & PM & PL \\
\hline PL & PM & PM & PM & PL & PL \\
\hline
\end{tabular}
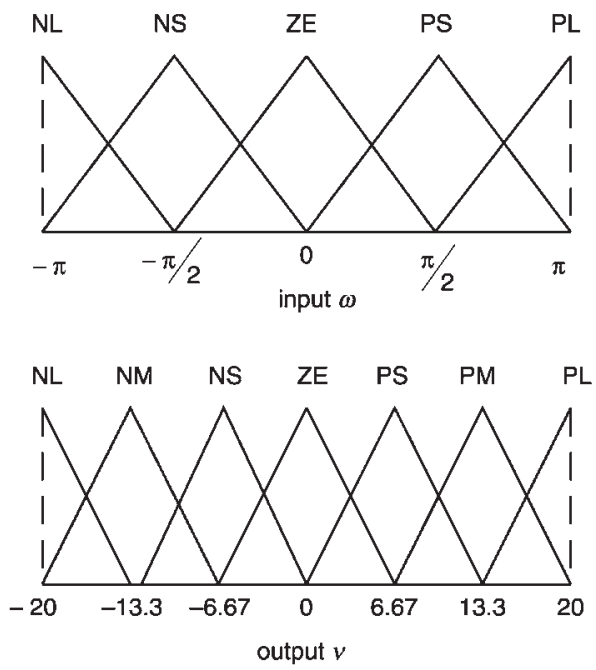

\begin{tabular}{|c|c|c|c|c|c|c|c|c|c|}
\hline$\theta$ & $-\pi$ & $-3 \pi / 4$ & $-\pi / 2$ & $-\pi / 4$ & 0 & $\pi / 4$ & $\pi / 2$ & $3 \pi / 4$ & $\pi$ \\
\hline$-\pi$ & -17.9 & -17.9 & -17.5 & -14.2 & -13.3 & -13.3 & -13.3 & -10.0 & -6.68 \\
\hline$-3 \pi / 4$ & -17.5 & -14.2 & -14.2 & -13.3 & -13.3 & -10.0 & -10.0 & -10.0 & -6.68 \\
\hline$-\pi / 2$ & -17.5 & -14.2 & -13.3 & -13.3 & -13.3 & -10.0 & -6.65 & -6.65 & -6.65 \\
\hline$-\pi / 4$ & -14.2 & -10.9 & -10.0 & -6.66 & -6.66 & -3.33 & 0.05 & 3.33 & 3.75 \\
\hline 0 & -13.3 & -10.0 & -6.66 & -3.33 & 0 & 3.33 & 6.66 & 10.0 & 13.3 \\
\hline$\pi / 4$ & -3.75 & -3.33 & -0.05 & 3.33 & 6.66 & 6.66 & 10.0 & 10.9 & 14.2 \\
\hline$\pi / 2$ & 6.65 & 6.65 & 6.65 & 10.0 & 13.3 & 13.3 & 13.3 & 14.2 & 17.5 \\
\hline $3 \pi / 4$ & 6.68 & 10.0 & 10.0 & 10.0 & 13.3 & 13.3 & 14.2 & 14.2 & 17.5 \\
\hline$\pi$ & 6.68 & 10.0 & 13.3 & 13.3 & 13.3 & 14.2 & 17.5 & 17.9 & 17.9 \\
\hline
\end{tabular}

$b$

Fig. 3 Equivalent fuzzy DPLL baseband model (a), and membership functions and fuzzy rule table (b) 
narrows the tracking loop bandwidth automatically when a receiver experiences significant acceleration.

In our FLC design, seven fuzzy terms are defined as the standard linguistic labels. These terms are: negative large (NL), negative medium (NM), negative small (NS), zero (ZE), positive small (PS), positive medium (PM) and positive large (PL). NL, NS, ZE, PS and PL are used over the domain of input variables $\left(\theta_{e, k}, \omega_{e, k}\right)$, and NL, NM, NS, ZE, PS, PM and PL are used over the domain of the output variable $\left(v_{k}\right)$. We choose triangular-shaped membership functions (MFs) for the input and output variables. The inference mechanism based on the Mamdani algorithm is utilised. The fuzzy rule table providing the human knowledge base of the controller is shown in Fig. $3 b$. It indicates that the absolute value of fuzzy output $v_{k}$ grows rapidly when the absolute phase error $\theta_{e, k}$ or frequency error $\omega_{e, k}$ becomes large, and improves the DPLL tracking capability. If the absolute phase error and frequency error are small, the fuzzy output is reduced, as is the loop bandwidth, which enhances the measurement accuracy. These fuzzy rules can be expressed as

$$
R^{i}: \text { If } x_{1} \text { is } A_{1}^{i} \text {, and } x_{2} \text { is } A_{2}^{i} \text {, then } y \text { is } B^{i} . i=1,2, \ldots, 25
$$

where $x_{1}, x_{2}$ and $y$ are linguistic variables, and $A_{1}^{i}, A_{2}^{i}$ and $B^{i}$ are linguistic labels (or fuzzy sets) characterised by MFs.

A defuzzification process is utilised to determine a crisp value according to the fuzzy output from the inference mechanism. The centre-average method, which calculates the centre of the area of the inference mechanism output possibility distribution, is used as the defuzzification strategy in our DPLL. The output $\bar{y}$ can be obtained as follows:

$$
\bar{y}=\frac{\sum_{i=1}^{n} y_{i} u\left(y_{i}\right)}{\sum_{i=1}^{n} u\left(y_{i}\right)}
$$

where $n$ is the number of fuzzy output sets, $y_{i}$ is the numerical value of the $i$ th output membership function, and $u\left(y_{i}\right)$ represents its membership value at the $i$ th quantisation level. When the fuzzy inputs $\theta_{e, k}$ and $\omega_{e, k}$ are close to zero in the steady state, it means that the phase lock status is accomplished and the FLC loop becomes a narrowband DPLL with the highest degree of measurement precision.

\section{Digital carrier loop design}

The order of a carrier loop is related to its capability to acquire different types of signal dynamics. Since a secondorder PLL is unconditionally stable but suffers from acceleration stress error, high-dynamic receivers typically use third- or fourth-order PLLs to provide the desirable characteristic of being able to track an accelerating frequency input. Stability becomes a major concern for these high-order loops, and analysis needs to be performed first in the analogue domain. The closed-loop system transfer functions with loop filters $\left(a \omega_{n} s+\omega_{n}^{2}\right) / s$, $\left(b \omega_{n} s^{2}+a \omega_{n}^{2} s+\omega_{n}^{3}\right) / s^{2}$ and $\left(c \omega_{n} s^{3}+b \omega_{n}^{2} s^{2}+a \omega_{n}^{3} s+\right.$ $\left.\omega_{n}^{4}\right) / s^{3}$ can be written as

$$
T(s)= \begin{cases}\frac{a \omega_{n} s+\omega_{n}^{2}}{s^{2}+a \omega_{n} s+\omega_{n}^{2}} & \text { (2nd order) } \\ \frac{b \omega_{n} s^{2}+a \omega_{n}^{2} s+\omega_{n}^{3}}{s^{3}+b \omega_{n} s^{2}+a \omega_{n}^{2} s+\omega_{n}^{3}} & \text { (3rd order } \\ \frac{c \omega_{n} s^{3}+b \omega_{n}^{2} s^{2}+a \omega_{n}^{3} s+\omega_{n}^{4}}{s^{4}+c \omega_{n} s^{3}+b \omega_{n}^{2} s^{2}+a \omega_{n}^{3} s+\omega_{n}^{4}} & \text { (4th order) }\end{cases}
$$

The stability condition can be determined using RouthHurtwitz criteria, and are found to be:

$$
\begin{array}{lr}
a, \omega_{n}>0 & \text { (2nd order) } \\
a, b, \omega_{n}>0 \text { and } a b>1 & \text { (3rd order) } \\
a, b, c, \omega_{n}>0, b c>a \text { and } a b c>a^{2}+c^{2} & \text { (4th order) }
\end{array}
$$

The frequency response of the tracking loop is obtained by substituting $s=j 2 \pi f$, where $f$ is the frequency in Hz. The single-sided loop noise bandwidth $B_{n}$ for the closed loop is defined by

$$
2 B_{n}=\int_{-\infty}^{+\infty}\left|T_{N}(j 2 \pi f)\right|^{2} d f
$$

with $\left|T_{N}(j 2 \pi f)\right|^{2}=T_{N}(j 2 \pi f) T_{N}(-j 2 \pi f)$

$$
\begin{aligned}
& T_{N}(j 2 \pi f) \\
& \quad=\frac{d_{1}(j 2 \pi f)^{N-1}+d_{2}(j 2 \pi f)^{N-2}+\cdots+d_{N}}{(j 2 \pi f)^{N}+d_{1}(j 2 \pi f)^{N-1}+d_{2}(j 2 \pi f)^{N-2}+\cdots+d_{N}}
\end{aligned}
$$

where $N$ is the order of transfer function. The analytical solution for the loop bandwidth can be computed as a function of $d_{1}, d_{2}, \cdots, d_{N}$ [8]. The results are summarised in Table 1 for loops of second- to fourth-order. The natural frequency can be found as:

Table 1: Loop noise bandwidth $B_{n}$ from loop constants

\begin{tabular}{llrl}
\hline & $T_{N}(s)=\frac{d_{1} s^{N-1}+d_{2} s^{N-2}+\cdots+d_{N}}{s^{N}+d_{1} s^{N-1}+d_{2} s^{N-2}+\cdots+d_{N}}$ & $2 B_{n}=\int_{-\infty}^{+\infty}|T(j 2 \pi f)|^{2} d f$ \\
\hline$N=2$ & $T_{2}(s)=\frac{a \omega_{n} s+\omega_{n}^{2}}{s^{2}+a \omega_{n} s+\omega_{n}^{2}}$ & $B_{n}=\frac{d_{1}^{2}+d_{2}}{4 d_{1}}=\left[\frac{1+a^{2}}{4 a}\right] \omega_{n}$ \\
$N=3$ & $T_{3}(s)=\frac{b \omega_{n} s^{2}+a \omega_{n}^{2} s+\omega_{n}^{3}}{s^{3}+b \omega_{n} s^{2}+a \omega_{n}^{2} s+\omega_{n}^{3}}$ & $B_{n}=\frac{d_{1}^{2} d_{2}-d_{1} d_{3}+d_{2}^{2}}{4\left(d_{1} d_{2}-d_{3}\right)}=\left[\frac{a b^{2}+a^{2}-b}{4(a b-1)}\right] \omega_{n}$ \\
$N=4$ & $T_{4}(s)=\frac{c \omega_{n} s^{3}+b \omega_{n}^{2} s^{2}+a \omega_{n}^{3} s+\omega_{n}^{4}}{s^{4}+c \omega_{n} s^{3}+b \omega_{n}^{2} s^{2}+a \omega_{n}^{3} s+\omega_{n}^{4}}$ & $B_{n}=\frac{d_{1}^{2} d_{2} d_{3}-d_{1} d_{3}^{2}-d_{1}^{3} d_{4}+d_{2}^{2} d_{3}-d_{1} d_{2} d_{4}-d_{3} d_{4}}{4\left(d_{1} d_{2} d_{3}-d_{3}^{2}-d_{1}^{2} d_{4}\right)}$ \\
& & $=\left[\frac{c^{2}(a b-c)+a\left(b^{2}-a c-1\right)-b c}{4\left(a c b-a^{2}-c^{2}\right)}\right] \omega_{n}$
\end{tabular}




$$
\omega_{n}= \begin{cases}{\left[\frac{4 a}{1+a^{2}}\right] B_{n}} & \text { (2nd order) } \\ {\left[\frac{4(a b-1)}{a b^{2}+a^{2}-b}\right] B_{n}} & \text { (3rd order) } \\ {\left[\frac{4\left(a b c-a^{2}-c^{2}\right)}{c^{2}(a b-c)+a\left(b^{2}-a c-1\right)-b c}\right] B_{n}} & \text { (4th order) }\end{cases}
$$

To build a digital PLL filter for software implementation, the backward Euler method (BEM, $1 / s \leftrightarrow T /\left(1-z^{-1}\right)$ ) with stable transformation is applied to mapping from $s$ to $z$. In this paper, $K_{d}$ denotes the gain of the phase detector chosen as unity for the arc-tangent type of discriminator, and $K_{n}$ is the gain of NCO. The mathematical model of the $\mathrm{NCO}$ can be expressed as

$$
N(z)=\frac{K_{n} z^{-1}}{1-z^{-1}}
$$

The digital filters have the following transfer functions:

$$
\begin{gathered}
F(z)=\frac{\left(K_{1}+K_{2}\right)-K_{1} z^{-1}}{1-z^{-1}} \quad \text { (2nd order) } \\
F(z)=\frac{\left(K_{1}+K_{2}+K_{3}\right)-\left(2 K_{1}+K_{2}\right) z^{-1}+K_{1} z^{-2}}{1-2 z^{-1}+z^{-2}} \text { (3rd order) } \\
F(z)=\frac{\left\{\begin{array}{c}
\left(K_{1}+K_{2}+K_{3}+K_{4}\right)-\left(3 K_{1}+2 K_{2}\right. \\
\left.+K_{3}\right) z^{-1}+\left(3 K_{1}+K_{2}\right) z^{-2}-K_{1} z^{-3}
\end{array}\right\}}{1-3 z^{-1}+3 z^{-2}-z^{-3}} \text { (4th order) }
\end{gathered}
$$

Substituting (15) and (16) into the carrier loops, the discretetime closed-loop transfer functions can be obtained,

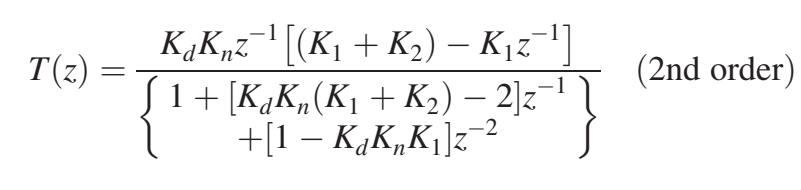

$$
\begin{aligned}
& T(z)= \\
& \frac{\left\{\begin{array}{c}
K_{d} K_{n} z^{-1}\left[\left(K_{1}+K_{2}+K_{3}\right)\right. \\
\left.-\left(2 K_{1}+K_{2}\right) z^{-1}+K_{1} z^{-2}\right]
\end{array}\right\}}{\left\{\begin{array}{c}
1+\left[K_{d} K_{n}\left(K_{1}+K_{2}+K_{3}\right)-3\right] z^{-1}+[3 \\
\left.-K_{d} K_{n}\left(2 K_{1}+K_{2}\right)\right] z^{-2}+\left[K_{d} K_{n} K_{1}-1\right] z^{-3}
\end{array}\right\}} \text { (3rd order) }
\end{aligned}
$$$$
\begin{aligned}
& T(z)= \\
& \left\{\begin{array}{c}
K_{d} K_{n} z^{-1}\left[\left(K_{1}+K_{2}+K_{3}+K_{4}\right)-\left(3 K_{1}+2 K_{2}\right.\right. \\
\left.\left.+K_{3}\right) z^{-1}+\left(3 K_{1}+K_{2}\right) z^{-2}-K_{1} z^{-3}\right]
\end{array}\right\} \\
& \left\{\begin{array}{c}
1+\left[K_{d} K_{n}\left(K_{1}+K_{2}+K_{3}+K_{4}\right)-4\right] z^{-1}+[6 \\
\left.-K_{d} K_{n}\left(3 K_{1}+2 K_{2}+K_{3}\right)\right] z^{-2}+\left[K _ { d } K _ { n } \left(3 K_{1}\right.\right. \\
\left.\left.+K_{2}\right)-4\right] z^{-3}+\left[1-K_{d} K_{n} K_{1}\right] z^{-4}
\end{array}\right\}
\end{aligned}
$$

with:

$$
\begin{gathered}
K_{1}=\frac{a \omega_{n} T}{K_{d} K_{n}}, K_{2}=\frac{\omega_{n}^{2} T^{2}}{K_{d} K_{n}} \quad(2 \text { nd order }) \\
K_{1}=\frac{b \omega_{n} T}{K_{d} K_{n}}, K_{2}=\frac{a \omega_{n}^{2} T^{2}}{K_{d} K_{n}}, K_{3}=\frac{\omega_{n}^{3} T^{3}}{K_{d} K_{n}} \quad \text { (3rd order) }
\end{gathered}
$$

$$
\begin{aligned}
& K_{1}=\frac{c \omega_{n} T}{K_{d} K_{n}}, K_{2}=\frac{b \omega_{n}^{2} T^{2}}{K_{d} K_{n}} \\
& K_{3}=\frac{a \omega_{n}^{3} T^{3}}{K_{d} K_{n}}, K_{4}=\frac{\omega_{n}^{4} T^{4}}{K_{d} K_{n}} \quad \text { (4th order) }
\end{aligned}
$$

where $K_{1}, K_{2}, K_{3}$ and $K_{4}$ are the digital filter coefficients derived from the BEM.

The systematic design procedure of the FLC carrier loop is shown in Fig. 4. The maximum velocity, acceleration and jerk stress of the users with GPS receivers need to be obtained first. We can then determine the desired PLL acquisition limitations, e.g. (a) lock range $\left(\omega_{D}\right),(b)$ frequency ramp range $\left(\alpha_{D}\right)$ and $(c)$ jerk range $\left(J_{D}\right)$ for a loop. A small tracking loop bandwidth is adopted to maintain accurate position and velocity measurements. After converting the analogue PLL to the digital domain via BEM, the fuzzy logic method is employed as an adaptive controller for the digital loop to perform bandwidth adjustment and rapid carrier phase tracking. The static and dynamic tests for different trajectories at a low CNR of $35 \mathrm{dBHz}$ are used to evaluate acquisition performance. Because the carrier loops are nonlinear near the threshold region, the Monte Carlo (MC) simulation should be employed to determine the true tracking capabilities of GPS receivers under various conditions. Through appropriate adjustment of the control rules and membership functions, an excellent FLC controller can be obtained with respect to desired tracking specifications.

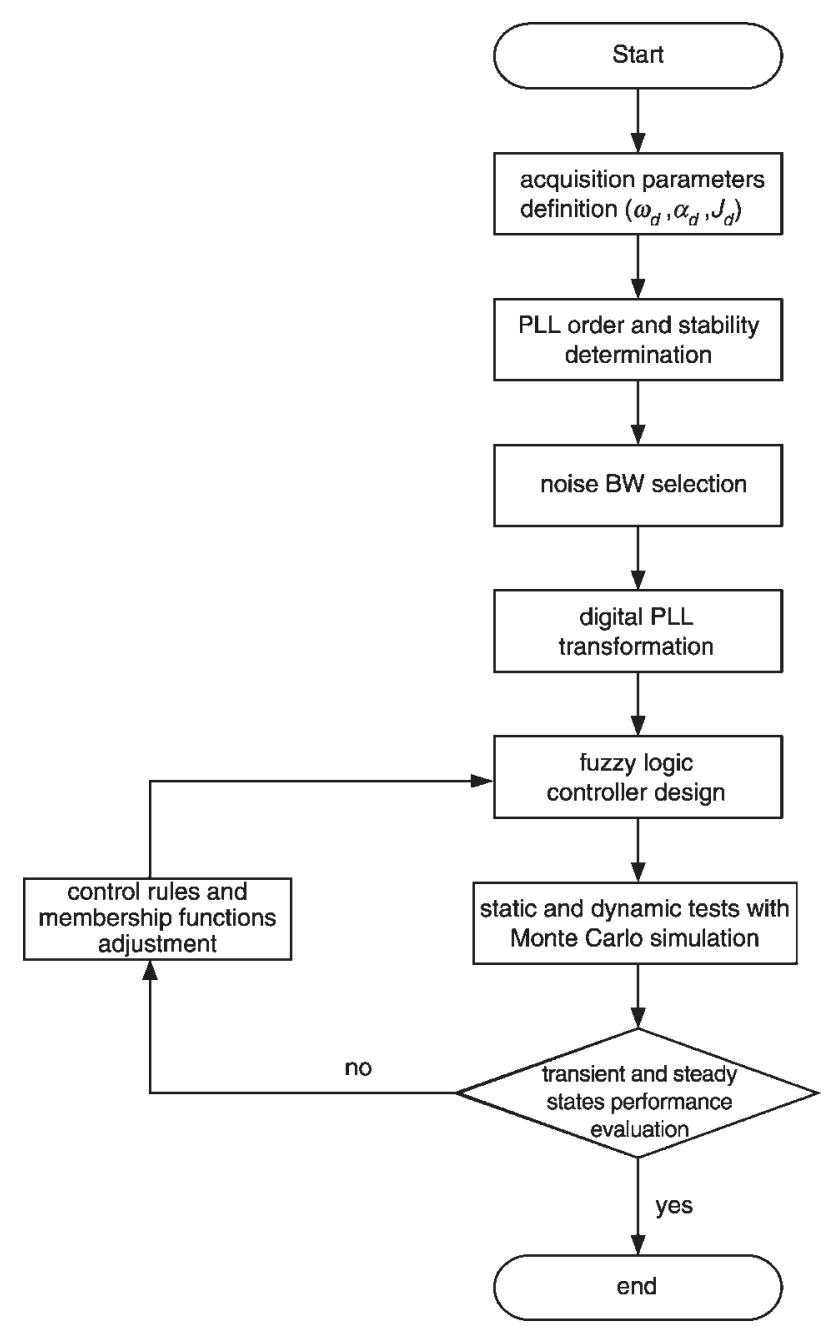

Fig. 4 Design procedure of intelligent carrier loop 


\section{Simulation results}

To demonstrate the performance of our proposed FLC GPS receiver, several computer simulation results under different circumstances will be presented. We consider tracking of the L1 carrier frequency in this work. The received signal is bandpass filtered, amplified and down-converted to IF and then digitised. The IF is fixed at $1.25 \mathrm{MHz}$, and a sampling frequency of $5 \mathrm{MHz}$ is selected. The CNR for GPS receiver is defined as

$$
C N R=10 \log (S N R \times B)=10 \log \left(\frac{P}{2 \times \sigma_{n}^{2} \times T}\right)[\mathrm{dBHz}]
$$

where $P$ is the power of the received signal, which can be assumed as unity without loss of generality (from 1 ), $\sigma_{n}^{2}$ is the power of AWGN and $T=1 \mathrm{~ms}$ is the PIT. Our DPLL tests here are conducted in a noisy condition with a lowest value of CNR $35 \mathrm{dBHz}$. The experiments are divided into two categories: $(a)$ a set of static tests, which are performed with constant phase, sinusoidal jitter, frequency and frequency ramp inputs, with each result obtained based on 1000 independent MC simulations, and $(b)$ the dynamic test, which is conducted with one simulated trajectory to evaluate the actual kinematic performance of the FLC loop. Four types of DPLL are compared, namely third-order DPLL with FLC, conventional second-, third- and fourthorder DPLLs. The complete fuzzy rules for the FL controller are illustrated in Fig. $3 b$. This controller is designed using two desired target operation limitations: a lock range of $150 \mathrm{~Hz}$ and a frequency ramp range of $412 \mathrm{~Hz} / \mathrm{s}$ (=8 $\mathrm{G}$ acceleration). For the five linguistic terms utilised for each input variable, 25 rules are developed for the FLC. The tracking loop bandwidths are set to $10 \mathrm{~Hz}$ and the corresponding digital filter parameters are summarised in Table 2.

\subsection{Static tests}

Figures $5 a, b$ illustrate the phase offset and phase error response of each of the DPLL schemes, when a $1 \mathrm{rad}$ step input is applied. The total simulation time is $200 \mathrm{~ms}$. It is seen that the FLC scheme can achieve a better performance with a settling time of $6 \mathrm{~ms}$ for $5 \%$ phase error specification. On the other hand, the conventional second-, third- and fourth-order loops require settling times of 229, 715 and $4389 \mathrm{~ms}$, respectively. Performances of each loop for phase jitter tracking are demonstrated in Fig. 6. A single-tone jitter with a frequency of $25 \mathrm{~Hz}$ and $A_{1}=1.2$ is considered. The residual phase errors of the traditional DPLLs are more than $0.5 \mathrm{rad}$ in the steady state. It is observed that the FLC loop can handle the tracking of the jitter waveform promptly in both amplitude and phase.

Figures $7 a, b$ show the system responses when an instantaneous $40 \mathrm{~Hz}$ frequency offset is applied. It can be seen that loss of lock occurs in traditional second- and

Table 2: Digital filter parameters of each DPLL

\begin{tabular}{ll}
\hline Type of DPLL & Digital filter parameters \\
\hline 2nd-order loop & $K_{1}=2.123 \times 10^{-5}, K_{2}=2.833 \times 10^{-7}$ \\
3rd-order loop & $K_{1}=3.434 \times 10^{-5}, K_{2}=1.422 \times 10^{-7}$ \\
& $K_{3}=1.648 \times 10^{-9}$ \\
4th-order loop & $K_{1}=1.882 \times 10^{-5}, K_{2}=1.888 \times 10^{-7}$ \\
& $K_{3}=9.1 \times 10^{-10}, \quad K_{4}=6.375 \times 10^{-12}$
\end{tabular}

$K_{d}=1, K_{n}=400 \pi, B_{n}=10 \mathrm{~Hz}$

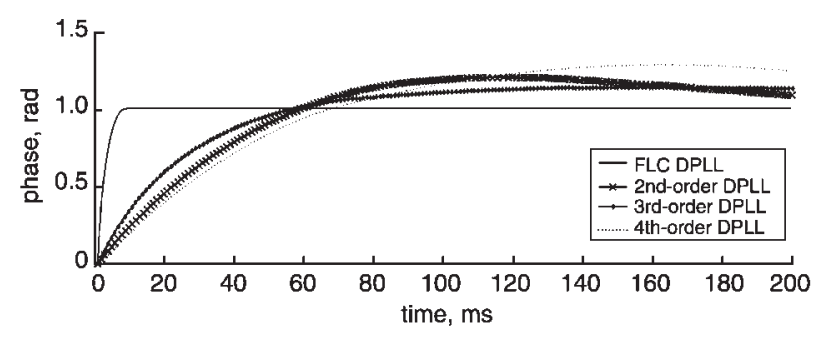

a

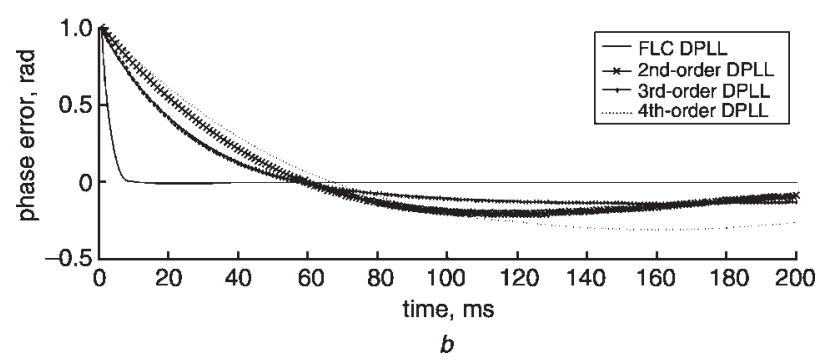

Fig. 5 Phase step responses and phase errors for each FLC, second-, third-and fourth-order DPLL when input phase offset is $1 \mathrm{rad}$

a Phase step response

$b$ Phase step error

third-order loops. The recovery process is rather slow, so a long pull-in time is required to regain lock. However, the phase tracking performance is excellent and the control loop remains locked the entire time with the FLC method. The FLC loop requires only $4 \mathrm{~ms}$ of settling time to reach the 5\% frequency error specification. Furthermore, an extended dynamic trajectory is also conducted with the input frequency of $200 \mathrm{~Hz}$. Figure 8 presents the loop tracking performance of the FLC scheme during the initial $200 \mathrm{~ms}$. The fuzzy algorithm works fairly well to accomplish a fast settling time of $110 \mathrm{~ms}$ for $5 \%$ frequency error specification. The overall performance comparisons of four kinds of DPLL are illustrated in Table 3, where input frequency offset varies from 20 to $200 \mathrm{~Hz}$. Note that, if no fuzzy controller is utilised, the capture ranges of traditional loops are rather small and result in loss of lock when the frequency

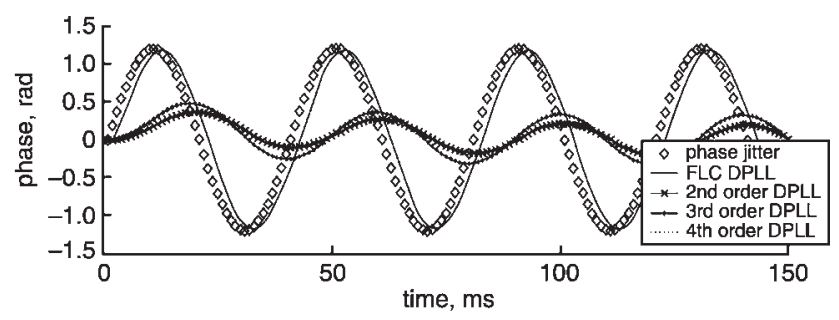

a

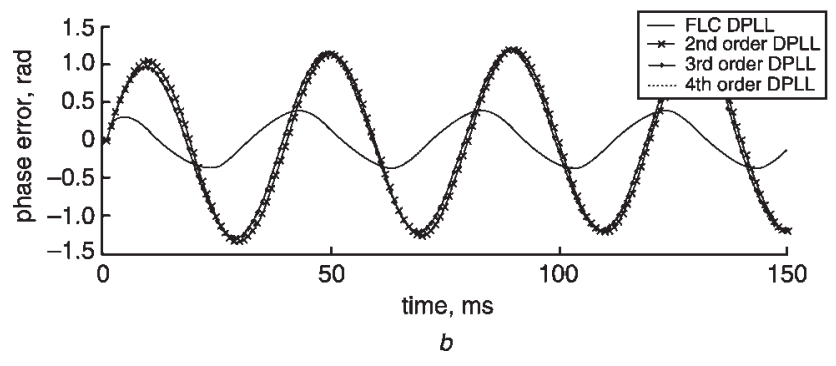

Fig. 6 Sinusoidal phase jitter responses and phase errors for each FLC, second-, third-and fourth-order DPLL when jitter frequency is $25 \mathrm{~Hz}$ with $A_{1}=1.2$

a Phase jitter response

$b$ Phase jitter error 
step is $>30 \mathrm{~Hz}$. As can been seen in Table 4, the FLC method can achieve better tracking performances with a $150 \mathrm{~Hz}$ lock range and a $400 \mathrm{~Hz}$ pull-in range. These results verify the effectiveness of the fuzzy method for wider lock range and rapid recovery from cycle skipping.
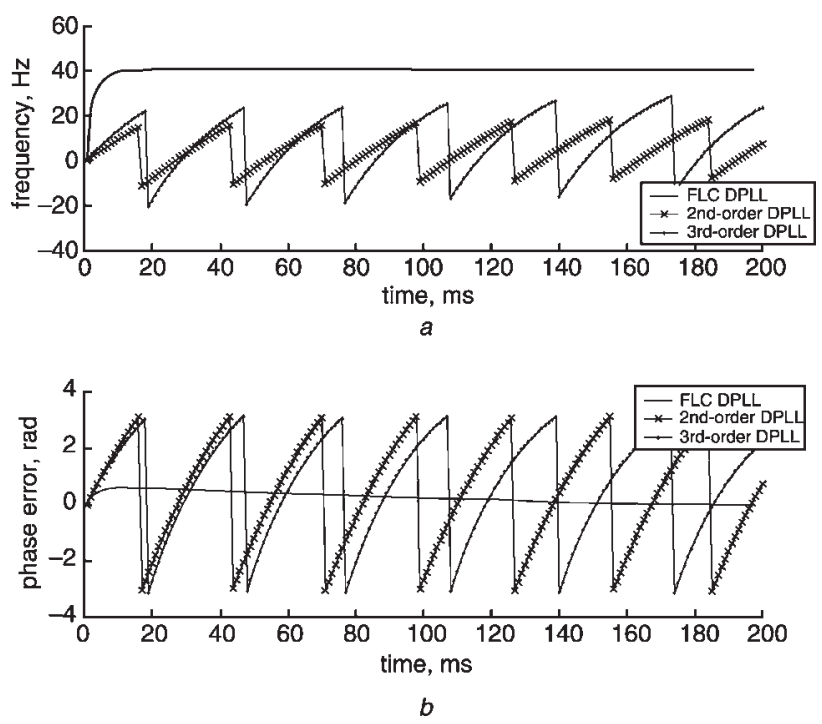

Fig. 7 Frequency step responses and phase errors for each $F L C$, second-and third-order DPLL when input frequency offset is $40 \mathrm{~Hz}$ $a$ Frequency step response

$b$ Phase error of frequency step
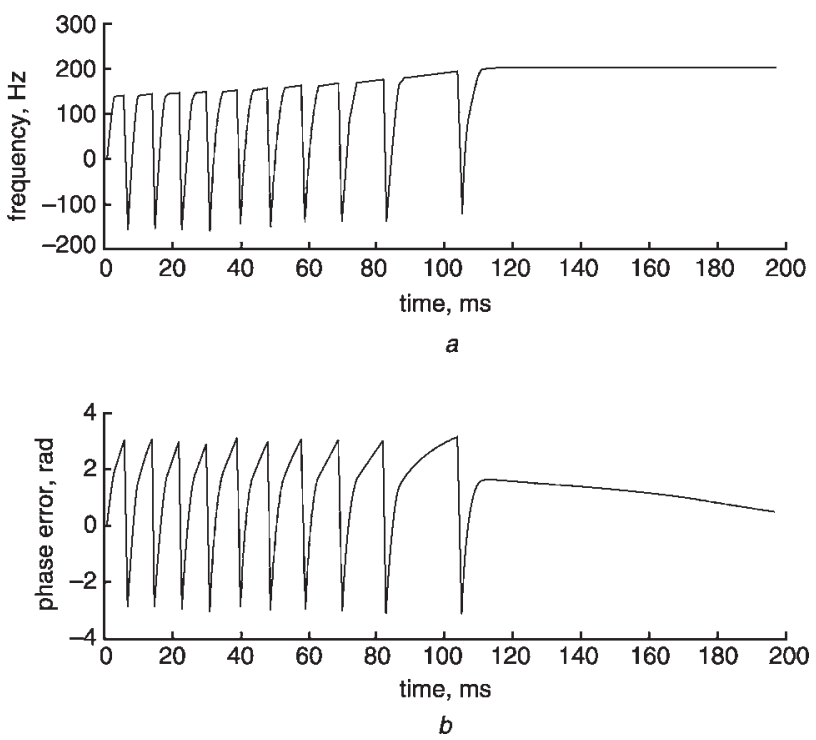

Fig. 8 Frequency step response and phase error for FLC DPLL when input frequency offset is $200 \mathrm{~Hz}$

$a$ Frequency step response

$b$ Phase error of frequency step
We show in Fig. 9 the simulation results of the FLC method under the severe kinematic environment, i.e. frequency ramp tracking. The rate of change of the Doppler frequency is set to be $400 \mathrm{~Hz} / \mathrm{s}$ as the dynamic input. The transient response of our method has significant improvement in the convergence rate to the zero steady state phase error. It is found that the FLC loop has a settling time of $14 \mathrm{~ms}$ for $5 \%$ frequency ramp error specification. Table 5 presents the settling time against vehicle dynamics for each scheme, in which the tests are conducted with different gravitational acceleration values ranging from $1 G$ to $8 G$. As can been seen, the conventional loops with narrow bandwidths suffer from smaller frequency ramp ranges

Table 4: Acquisition limitations for each DPLL scheme

\begin{tabular}{|c|c|c|c|}
\hline Type of DPLL & $\begin{array}{l}\text { Lock } \\
\text { range, } \mathrm{Hz}\end{array}$ & $\begin{array}{l}\text { Pull-in } \\
\text { range, } \mathrm{Hz}\end{array}$ & $\begin{array}{l}\text { Freq. ramp } \\
\text { range, } \mathrm{Hz} / \mathrm{s}\end{array}$ \\
\hline $\begin{array}{l}\text { Proposed FLC } \\
\text { loop }\end{array}$ & 150 & 400 & $\begin{array}{l}411.7 \\
(8 G)\end{array}$ \\
\hline $\begin{array}{l}\text { Conventional } \\
\text { 2nd-order loop }\end{array}$ & 20 & 70 & $\begin{array}{l}164.8 \\
(3.2 G)\end{array}$ \\
\hline $\begin{array}{l}\text { Conventional } \\
\text { 3rd-order loop }\end{array}$ & 26 & 95 & $\begin{array}{l}221.5 \\
(4.3 G)\end{array}$ \\
\hline $\begin{array}{l}\text { Conventional } \\
\text { 4th-order loop }\end{array}$ & 16 & 18 & $\begin{array}{l}118.5 \\
(2.3 G)\end{array}$ \\
\hline
\end{tabular}

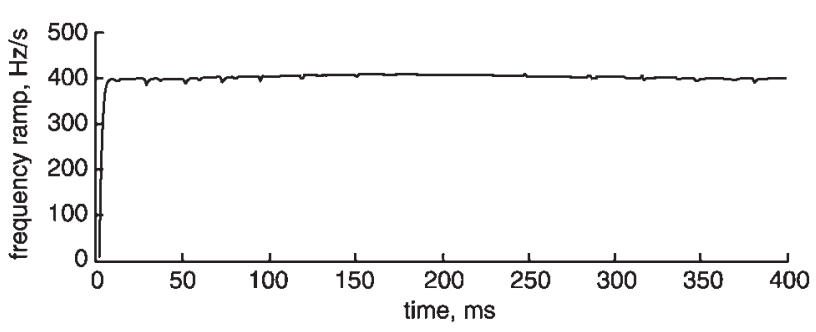

a

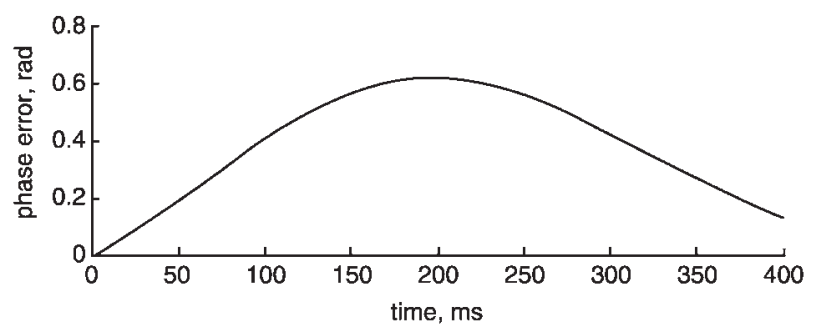

$b$

Fig. 9 Frequency ramp response and phase error for FLC DPLL when input frequency ramp is $400 \mathrm{~Hz} / \mathrm{s}$

a Frequency ramp response

$b$ Phase error of frequency ramp

Table 3: Settling times for each DPLL scheme in Doppler frequency shifted environments

\begin{tabular}{|c|c|c|c|c|c|c|c|c|c|c|}
\hline Frequency step, $\mathrm{Hz}$ & 20 & 40 & 60 & 80 & 100 & 120 & 140 & 160 & 180 & 200 \\
\hline Type of DPLL & \multicolumn{10}{|c|}{ Settling time, ms } \\
\hline Proposed FLC loop & 3 & 4 & 5 & 6 & 7 & 8 & 41 & 57 (CS) & 82 (CS) & $110(\mathrm{CS})$ \\
\hline Conventional 2nd-order loop & 229 & 931 (CS) & 2293 (CS) & LL & LL & LL & LL & LL & LL & LL \\
\hline Conventional 3rd-order loop & 717 & 890 (CS) & 1132 (CS) & 1462 (CS) & LL & LL & LL & LL & LL & LL \\
\hline Conventional 4th-order loop & LL & LL & LL & LL & LL & LL & LL & LL & LL & LL \\
\hline
\end{tabular}

CS: cycle slipping; LL: loss of lock 
Table 5: Settling times for each DPLL scheme in Doppler frequency ramp environments

\begin{tabular}{|c|c|c|c|c|c|c|c|c|}
\hline Gravitational acceleration, G & 1 & 2 & 3 & 4 & 5 & 6 & 7 & 8 \\
\hline Freq. ramp offset, $\mathrm{Hz} / \mathrm{s}$ & 51.5 & 102.9 & 154.4 & 205.9 & 257.3 & 308.8 & 360.2 & 411.7 \\
\hline Type of DPLL & \multicolumn{8}{|c|}{ Settling time, ms } \\
\hline Proposed FLC loop & 7 & 7 & 8 & 8 & 10 & 12 & 13 & 15 \\
\hline Conventional 2nd-order loop & 228 & 230 & 233 & LL & LL & LL & LL & LL \\
\hline Conventional 3rd-order loop & 715 & 721 & 725 & 982 (CS) & LL & LL & LL & LL \\
\hline Conventional 4th-order loop & 4341 & 4344 & LL & LL & LL & LL & LL & LL \\
\hline
\end{tabular}

CS: cycle slipping; LL: loss of lock

(approximately $2 \sim 4 G$ ). This difficulty can be resolved by incorporating a fuzzy controller into the loop, and the corresponding tracking capability can be extended to $8 \mathrm{G}$. It is obvious that our proposed method outperforms the traditional DPLLs with a faster transient response and a larger frequency ramp tracking range.

\subsection{Dynamic test}

The results of the dynamic measurements are shown in Fig. 10. Here a high performance aircraft carrying a GPS receiver is moving at a 500 miles/h speed directly toward the satellite for the initial $10 \mathrm{~s}$. Therefore, the airplane's trajectory includes positive and negative acceleration pulses of $10 \mathrm{~s}$ duration with $7 \mathrm{G}$ magnitude, which are separated by $10 \mathrm{~s}$ of constant velocity. The velocity track can be scaled to an equivalent Doppler frequency trajectory using a conversion factor of $5.2514 \mathrm{~Hz} \mathrm{~s} / \mathrm{m}$ for the $\mathrm{L} 1$ signal. As shown in the Figures, the corresponding Doppler frequency offset varies between $1.714 \mathrm{MHz}$ and $4.774 \mathrm{MHz}$ in this mobile condition. The FLC loop is capable of tracking the

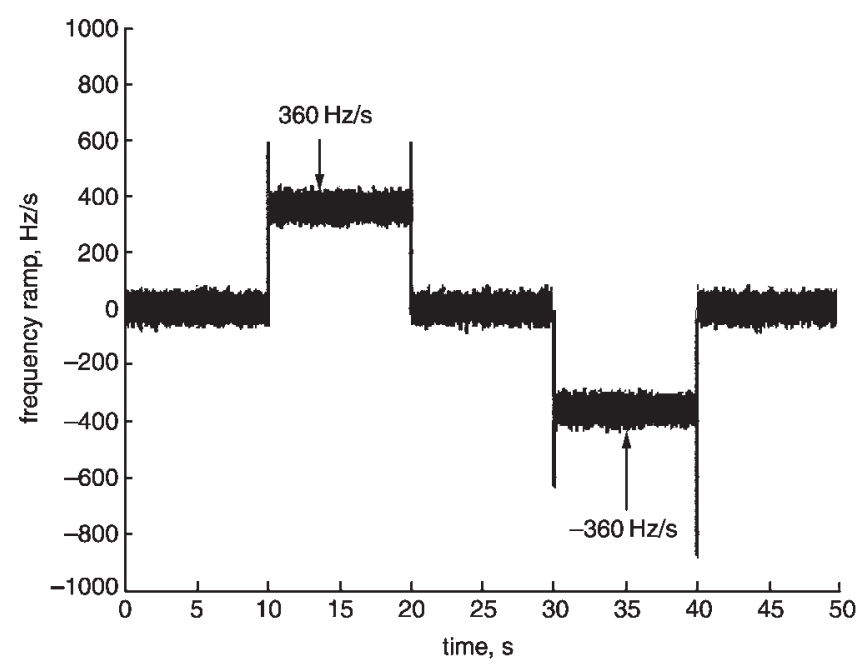

a

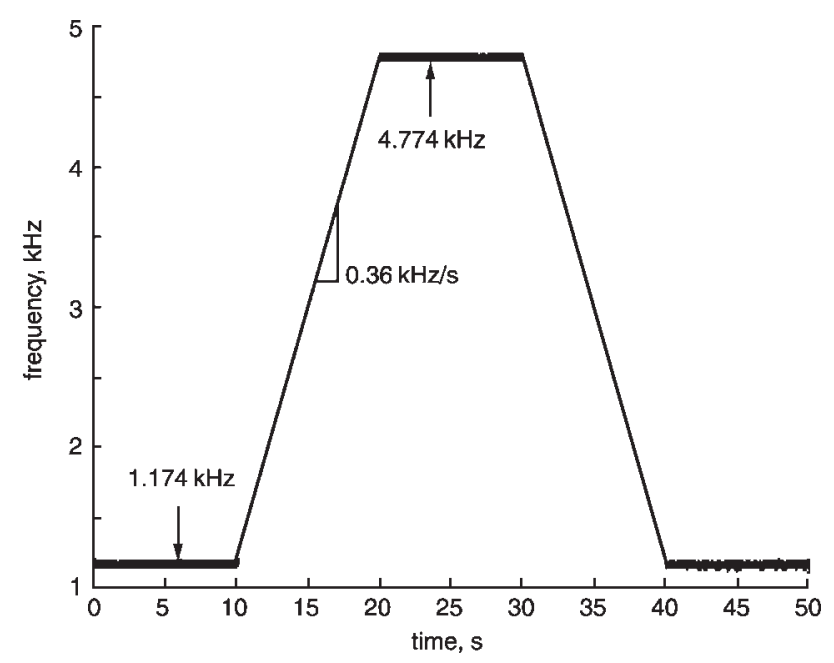

$b$

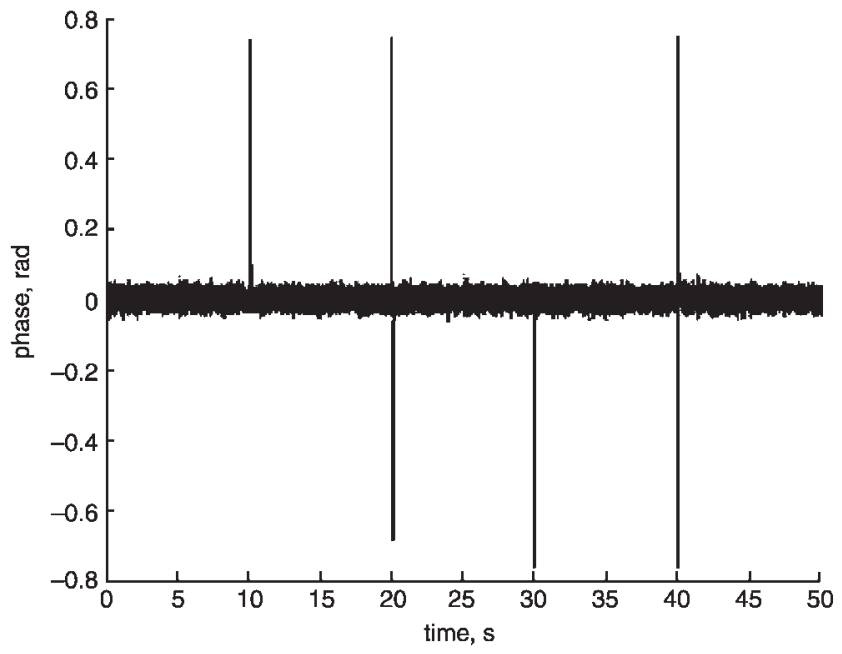

Fig. 10 Dynamic test results using FLC carrier loop

$\mathrm{CNR}=35 \mathrm{dBHz}$

$a$ Frequency ramp response

$b$ Frequency response

$c$ Phase error response 
acceleration carrier signal $( \pm 360 \mathrm{~Hz} / \mathrm{s})$ without losing lock in the transient state. The benefit of this fuzzy receiver is that a fast convergence and broader frequency locking range can be achieved in kinematic conditions. Moreover, it also has the capacity to recover quickly from loss-of-lock conditions during high-dynamic stress periods.

\section{Conclusions}

In this paper, a new method for improving the phase tracking performance of GPS receivers has been presented. Under a wide variety of environmental conditions, the proposed FLC scheme can control the DPLL robustly and vary the loop bandwidth adaptively during the dynamic periods. A step-by-step intelligent carrier loop design solution is developed to obtain stable and reliable operation in a diversity of kinematic trajectories. In addition, mathematical derivation of digital filters based on backward Euler transformation has been performed from second- to fourth-order loops. Numerical experiments on the FLC third-order DPLL and conventional loops are compared for both static and dynamic tests at a lowest CNR of $35 \mathrm{dBHz}$. Simulation results indicate that our FLC carrier loop is capable of rapider tracking and exhibits wider lock range than conventional loops. On the basis of loop bandwidth accommodation, the FLC method achieves three superior acquisition characteristics: (i) a lock range of $150 \mathrm{~Hz}$ and pull-in range of $400 \mathrm{~Hz}$; (ii) a more rapid pull-in process; and (iii) a frequency ramp range of $412 \mathrm{~Hz} / \mathrm{s} \quad(8 G$ acceleration) for the L1 carrier. Moreover, our simple and flexible fuzzy design can be incorporated into microcomputer software for very cost-effective implementation.

\section{References}

1 Simon, D., and El-Sherief, H.: 'Fuzzy logic digital phase-locked loop filter design', IEEE Trans. Fuzzy Syst., 1995, 3, pp. 211-218

2 Jwo, D.J.: 'Optimisation and sensitivity analysis of GPS receive tracking loops in dynamic environments', IEE Proc., Radar Sonar Navig., 2001, 148, pp. 241-250

3 Best, R.E.: 'Phase-locked loops, theory, design and applications' (McGraw-Hill, Inc., 1993)

4 Tsui, J.B.Y.: 'Fundamentals of global positioning system receivers, a software approach' (John Wiley \& Sons, Canada, 2000)

5 Braasch, M.S., and Van Dierendonck, A.J.: 'GPS receiver architectures and measurements', Proc. IEEE, 1999, 87, pp. 48-64

6 Stephens, D.R.: 'Phase-locked loops for wireless communications: digital and analog implementation' (Prentice Hall, Kluwer Academic 1998)

7 Kaplan, E.D.: 'Understanding GPS: principles and application' (Artech House, London, 1996)

8 Stephen, S.A., and Thomas, J.B.: 'Controlled-root formulation for digital phase-locked loops', IEEE Trans. Aerosp. Electron. Syst., 1995, 31 pp. $78-95$ 